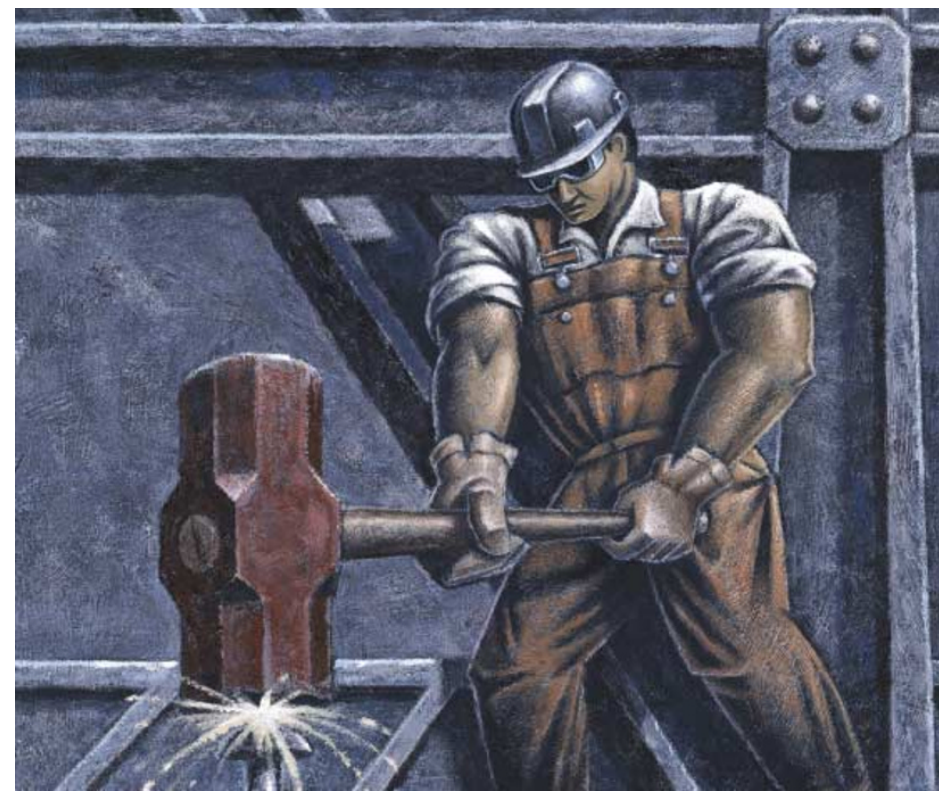

SYSTEMS BIOLOGY

\title{
A powerful combination
}

Reporting in Molecular Cell, Marc Vidal and colleagues show, for the first time, how combining two established techniques - double-genetic-perturbation genetic analysis and large-scale protein interaction (interactome) mapping strategies, which identify functional and physical interactions, respectively - can help us to understand biological processes at a systems level in a metazoan organism.

The authors wanted to identify components of the human TGF $\beta$ signalling pathway, to try and better understand TGF $\beta$ signalling and its dysregulation in disease. As many of the known TGF $\beta$-signalling-pathway components are highly conserved, they chose to study the Caenorhabditis elegans DAF7/TGF $\beta$ pathway.

First, Vidal and co-workers carried out a large-scale yeast two-hybrid (Y2H) screen using 6 known components of the DAF7/TGF $\beta$ pathway as bait. Of the 28 potential interacting proteins that were identified, 19 were used as bait in a second $\mathrm{Y} 2 \mathrm{H}$ screen. In total, 71 interactions and 59 potential interacting proteins were identified, and over half of these proteins have strong sequence similarity to human proteins. The physical interactions of the interactome map were then validated using co-affinity purification assays.

To find out about the functional relationships in this pathway, Vidal and colleagues used RNA interference (RNAi) to knock down genes that were derived from the interactome map in various loss-of-function mutant backgrounds that correspond to known DAF7/TGF $\beta$-pathway components. This double genetic perturbation identified 13 genetic interactions among 5 of the known DAF7/TGF $\beta$-pathway genes and 9 of the genes that were derived from the interactome map.

The authors went on to characterize one of these genes (W01G7.1) and identified it as daf-5. They suggest that W01G7.1/DAF5 is the C. elegans homologue of human SNO/SKI, which functions in the mammalian TGF $\beta$ pathway. The remaining 8 proteins are not known to have a role in TGF $\beta$ signalling in any species.

As both RNAi and $\mathrm{Y} 2 \mathrm{H}$ have their shortcomings, and generate a number of false-negative results, the authors caution that there are likely to be many more genes and interactions involved in the DAF7/TGF $\beta$ pathway than were identified by this study. Nevertheless, this study does reveal that the DAF7/TGF $\beta$ pathway is more complex than present models indicate.

The future of this combined approach looks promising. As the C. elegans genome-scale geneknockout project progresses and more genetic mutants become available, more in-depth functional analyses could be possible and the power of this combined approach should only increase.

Natalie Wilson

\section{d0) References and links} ORIGINAL RESEARCH PAPER Tewari, M. et al. Systematic interactome mapping and genetic perturbation analysis of a $C$. elegans TGF- $\beta$ signaling network. Mol. Cell 13, 469-482 (2004)
H I G H L I G H T S

\section{IN BRIEF}

RNA

Sequence-specific inhibition of small RNA function.

Hutvágner, G. et al. PLoS Biology 24 Feb 2004 (doi:10.1371/journal.pbio.0020098)

The authors show that 2'-O-methyl oligonucleotides that are complementary to small RNAs can bind to the RNA-induced silencing complex, and function as potent and irreversible inhibitors of RNA silencing in vivo and in vitro. As these oligonucleotides block the function of siRNAs and microRNAs in vivo, they will be useful for the functional characterization of the many small RNAs that have been identified recently. Furthermore, tethered oligonucleotides can be used to identify small-RNA-associated proteins, which might lead to new insights into the RNAi pathway.

\section{CELL CYCLE}

Recovery from DNA damage checkpoint arrest by PP1-mediated inhibition of Chk1.

den Elzen, N. R. \& O'Connell, M. J. EMBO J. 23, 908-918 (2004)

The G2 DNA-damage checkpoint prevents mitotic entry in the presence of DNA damage, but how the checkpoint is released and cell-cycle re-entry occurs is poorly understood. den Elzen and O'Connell have now identified the type-I protein phosphatase Dis2 as a key component of a checkpoint-release pathway in fission yeast. Dis2 specifically dephosphorylates, and thereby inactivates, the checkpoint-effector protein Chk1 kinase - but does not affect the function or phosphorylation of other checkpoint proteins.

\section{STEM CELLS}

Derivation of embryonic stem-cell lines from human blastocysts.

Cowan, C. A. et al. N. Engl. J. Med. 3 March 2004 (doi:10.1056/NEJMsr040330)

A group led by Douglas Melton has derived and characterized 17 new human embryonic stem-cell lines that meet strict criteria for easy and routine in vitro cell culture. These cell lines are available to scientists with a Material Transfer Agreement, but only for noncommercial research purposes. Under current regulations, the cell lines cannot be used for research that is funded by NIH funds. Information on how to obtain the cells and detailed protocols are available at http://www.mcb.harvard.edu/melton/hues.

\section{MOLECULAR MOTORS}

A kinesin-like motor inhibits microtubule dynamic instability.

Bringmann, H. et al. Science 303, 1519-1522 (2004)

The Xenopus laevis kinesin-like protein 1 (Xklp1) is important for chromosome-microtubule interactions and spindle-pole extension. Bringmann et al. now show that Xklp1 combines two properties that are characteristic of different classes of kinesin-like proteins: it is a fast, non-processive, plus-end-directed motor and inhibits microtubule polymerization and depolymerization.

The inhibitory effect is mediated by a global structural change in the microtubule lattice that is induced by the presence of bound Xklp1. 